\title{
Enhancing Quantitative Precipitation Estimation Over the Continental United States Using a Ground-Space Multi-Sensor Integration Approach
}

\author{
Qing Cao, Member, IEEE, Yixin Wen, Yang Hong, Jonathan J. Gourley, and Pierre-Emmanuel Kirstetter
}

\begin{abstract}
Quantitative precipitation estimation (QPE) based on ground weather radar could be considerably affected by the broadening, ascent, and blockage of the radar beam. These problems are particularly prevalent in mountainous regions. The current study proposes a multi-sensor approach to improve the ground-radar QPE in complex terrain. The proposed method, namely the Vertical Profile of Reflectivity (VPR) Identification and Enhancement (VPR-IE), integrates NOAA's National Mosaic QPE (NMQ) system and NASA's Tropical Rainfall Measuring Mission (TRMM) precipitation radar (PR) measurements. This study demonstrates promising performance of VPR-IE in the Mountainous West Region of the U.S. The potential error sources of this approach and its real-time implementation over the Continental United States are addressed as well.
\end{abstract}

Index Terms-Quantitative precipitation estimation (QPE), satellite remote sensing, vertical profile of reflectivity (VPR), weather radar.

\section{INTRODUCTION}

$\mathbf{T}$ HE operational U.S. Next-Generation Radar network (NEXRAD) provides high-resolution precipitation measurements over the Continental United States (CONUS), which are essential for the warning and forecasts of hazardous weather and flash floods. However, NEXRAD has inherent limitations in the low-level surveillance of the atmosphere [1]. Earth's curvature causes the radar to sample higher in the atmosphere

Manuscript received January 29, 2013; revised July 7, 2013 and October 2, 2013; accepted November 11, 2013. This work was supported by the National Aeronautics and Space Administration under Grant NNX11AL78G with the title of "Incorporating NASA Spaceborne Precipitation Research Products into National Mosaic QPE Real-time System for Improved Short-term Weather Prediction at Colorado Basin River Forecast Center".

Q. Cao and Y. Wen are with the Hydrometeorology \& Remote Sensing Laboratory and the Advanced Radar Research Center, University of Oklahoma, Norman, OK 73072 USA.

Y. Hong is with the Hydrometeorology \& Remote Sensing Laboratory and the Advanced Radar Research Center, University of Oklahoma, Norman, OK 73072 USA and also with the Department of Hydraulic Engineering, Tsinghua University, Beijing 100084, China (e-mail: yanghong@ou.edu).

J. J. Gourley and P.-E. Kirstetter are with the Hydrometeorology \& Remote Sensing Laboratory, University of Oklahoma, Norman, OK 73072 USA and also with NOAA's National Severe Storms Laboratory, Norman, OK 73072 USA.

Color versions of one or more of the figures in this paper are available online at http://ieeexplore.ieee.org.

Digital Object Identifier 10.1109/LGRS.2013.2295768 with increasing range, and partial or even complete beam blockages often exist in complex terrain. Given these limitations, near-surface measurements, which are desirable for accurate quantitative precipitation estimation (QPE), are only obtained within limited regions. At far range and in shielded regions, especially for the Mountainous West Region (MW-Region) of the U.S., NEXRAD measurements may potentially have large errors for QPE.

The vertical profile of radar reflectivity (VPR) provides valuable microphysical information linking the radar measurements aloft to the near-surface precipitation [2], [3]. Studies have shown that a representative VPR is useful to improve the ground-radar QPE by mitigating bright band (BB) contamination and radar beam overshooting [4]-[12]. These studies, however, have mainly relied on ground-radar-derived VPRs, which would be degraded by the effects of radar beam broadening and beam blockage. Recently the research group at the University of Oklahoma (OU) has investigated the vertical structure of precipitation using spaceborne radar [3], [13], [14], i.e., precipitation radar (PR) onboard the National Aeronautics and Space Administration (NASA)'s Tropical Rainfall Measuring Mission (TRMM) satellite [15], [16]. Given the fact that PR provides beam-blockage-free and a seamless coverage of precipitation measurements, those studies suggested a novel approach of integrating ground-radar and spaceborne radar for QPE. A concept of QPE enhancement, namely the VPR Identification and Enhancement (VPR-IE), has been proposed for such purposes [13]. This concept was only tested using a simple VPR correction for a single PR pass in our previous study.

The current study proposes an enhanced VPR-IE scheme, which applies both climatological and storm-scale VPR models to improve ground-radar QPE. The proposed VPR-IE scheme integrates the National Mosaic and Multi-sensor QPE (NMQ) system [17] and NASA's TRMM-PR products. A real-time system of this VPR-IE scheme is being constructed at OU and is expected to provide near-real-time, CONUS-wide, enhanced QPE products to users and researchers in the radar, satellite, meteorology and hydrology communities. This letter gives a detailed description of the proposed real-time VPR-IE system, with performance evaluation in AZ where NEXRAD radar QPE is typically degraded by the lack of near-surface radar observations. The system's potential error sources and its realtime implementation over the CONUS are discussed as well. 


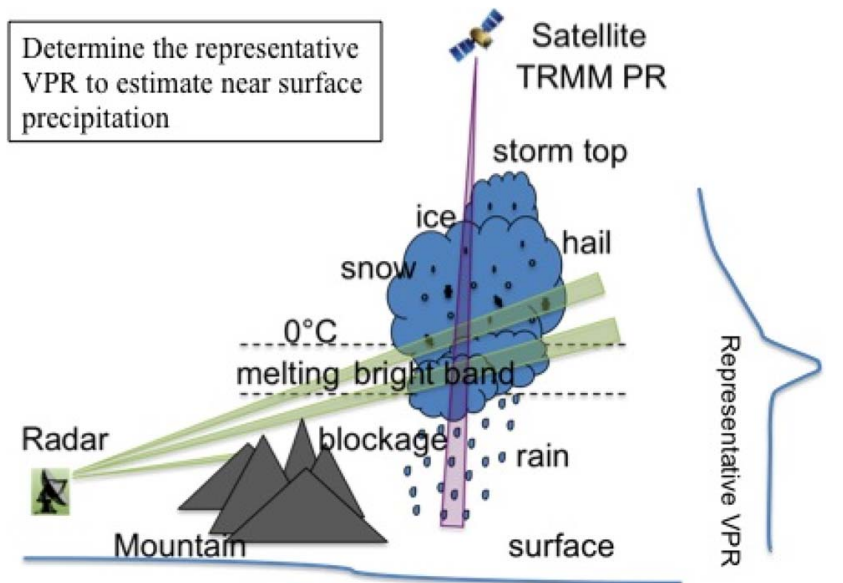

Fig. 1. Illustration of using spaceborne radar measured representative VPR to improve the near-surface QPE based on ground radar.

\section{Vertical Structure of Precipitation}

Different physical processes are associated with the state of hydrometeors at various heights in a precipitation system (Fig. 1). Solid particles above the $0{ }^{\circ} \mathrm{C}$ isotherm normally give a radar reflectivity much lower than liquid drops (below the melting layer) for the same water content. On the other hand, the melting layer is typically associated with BB signals (enhanced radar reflectivity). Therefore, overshooting (or intercepting) the melting layer by the radar beam may lead to an underestimation (or overestimation) of the near-surface precipitation. Given beam blockages in mountainous regions, this kind of overshooting (or intercepting) is common for ground radars.

As illustrated in Fig. 1, the VPR measured by spaceborne radar reveals the complete vertical structure of precipitation, linking ground-radar measurements aloft to the near-surface precipitation. Given a representative VPR, the ground-radar QPE can certainly be improved by considering the vertical structure of hydrometeor phase. This is the basis of the VPRIE method. Correspondingly, characterizing the representative VPR is one of the major tasks for VPR-IE.

\section{PRoposed VPR-IE SCHEME}

Fig. 2 shows the framework of the proposed real-time VPRIE system. The VPR-IE system incorporates NMQ and TRMM products in real-time through the links connecting data servers at the National Severe Storms Laboratory (NSSL) and NASA Precipitation Processing Systems (PPS). As for VPR correction, 3-D radar mosaic (S-band 3-D reflectivity) and TRMMPR products (2A25 and 2A23) are the primary inputs. 2A25 includes PR-measured Ku-band 3-D reflectivity. 2A23 provides the precipitation type identification (stratiform or convective), detection of the melting layer, and quantities such as the height of BB peak, the height of freezing level, etc. The improved near-surface QPE is evaluated by rain gauge measurements and the results are provided to users via a web-based data delivery system. The VPR correction is a major component of VPR-IE system. More details of the methodology are given in following sub-sections.

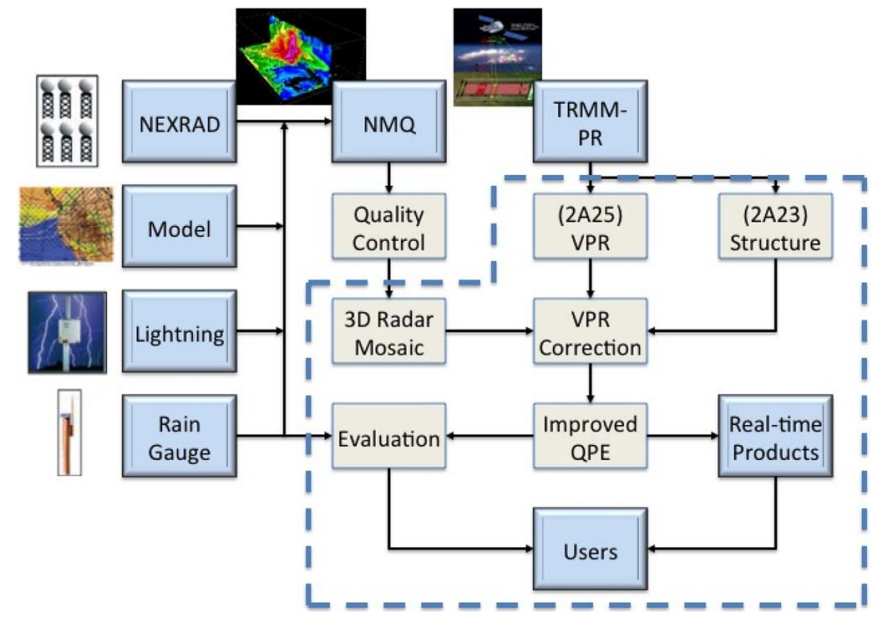

Fig. 2. Framework of proposed VPR-IE system at OU. The major components associated with VPR correction are enclosed by dashed lines.

\section{A. Ku-Band to S-Band Conversion}

The scattering characteristics of hydrometeors at different frequencies may lead to different VPRs. In order to use Ku-band VPR to correct S-band VPR, a conversion from Ku-band to S-band should be applied. Our previous works have introduced two approaches: VPR model approach [13] and empirical conversion [14].

Wen et al. [13] have presented a physically-based VPR model, which assumes five parameters (cloud top, freezing level height, melting layer width, rain region VPR slope, and ice density factor) to compute a VPR. The model parameters are fitted from observed VPR using nonlinear regression. This method is computationally expensive for real-time data processing.

Cao et al. [14] have derived a set of empirical relations for hydrometeors of different types or phases (snow, ice/hail, raindrop, melting particle). Radar dual-frequency ratio (DFR, unit in $\mathrm{dB}$ ), which links Ku-band and S-band reflectivity (Z, unit in dBZ), can be computed from Ku-band reflectivity using polynomial relations. Coefficients $\mathrm{a}_{0} \ldots \mathrm{a}_{4}$ have different values, depending on the type and phase of hydrometeors

$$
\begin{aligned}
Z(\mathrm{~S})= & Z(\mathrm{Ku})+\mathrm{DFR} \\
\mathrm{DFR}= & a_{0}+a_{1} Z(\mathrm{Ku})+a_{2} Z(\mathrm{Ku})^{2} \\
& +a_{3} Z(\mathrm{Ku})^{3}+a_{4} Z(\mathrm{Ku})^{4} .
\end{aligned}
$$

The empirical conversion is computationally efficient and easily implemented. The detailed conversion procedure and the coefficients in empirical relations can be found in [14].

\section{B. Apparent VPR at Different Radar Ranges}

VPR measurements from ground radar may be degraded (i.e., having a worse vertical resolution) by the beam broadening effect, especially at far range [6]-[9]. Smoothing of the VPR may lead to an incorrect quantification of physical processes. The smoothed VPR is normally regarded as the apparent VPR (AVPR) [9]. Generally, VPR measurements from TRMM-PR are unaffected by the beam broadening effect in the vertical direction [3]. The derived S-band VPR from TRMM-PR measurements (as addressed in Section II-A) should be converted 

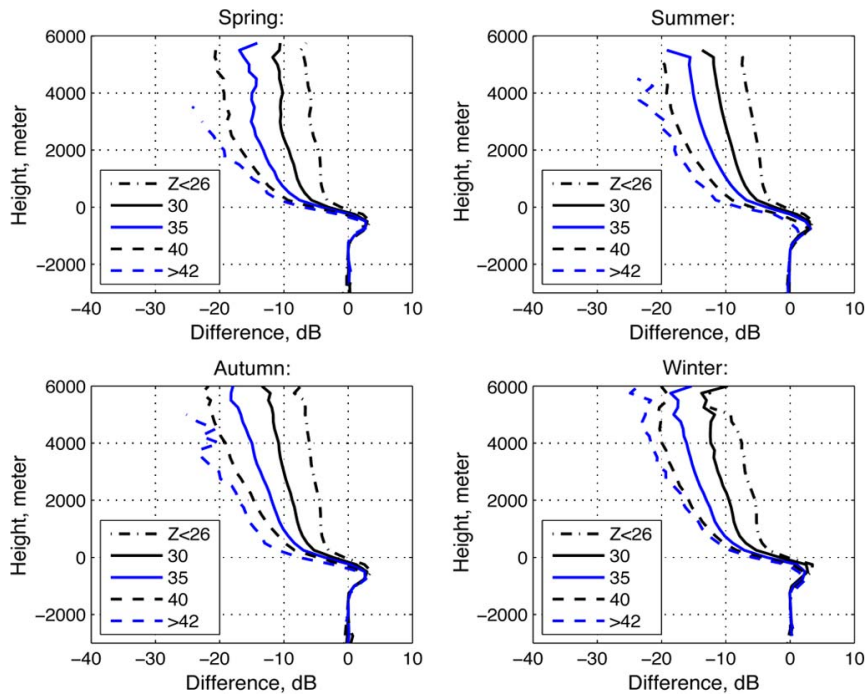

Fig. 3. S-band climatological VPRs. Y-axis denotes the height relative to the freezing level.

to the AVPR to match ground-radar measurements at different radar ranges [9], [13]. To obtain the AVPR, a Gaussian function is used by VPR-IE to smooth the representative VPR. Given a specific radar range, the width of the Gaussian function is determined by the 3 -dB radar beam width.

\section{Climatological VPRs for VPR Correction}

The proposed VPR-IE system applies climatological VPRs derived from long-term PR observations [3] to correct the radar QPE that has been degraded. Using the empirical conversion approach in [14], the VPR statistics in [3] have been repeated to obtain the S-band climatological VPRs for the proposed VPRIE system (as shown in Fig. 3). The climatological VPR $z(h)$ is represented by the ratio of VPR to the reflectivity value at a reference height $h_{0}$

$$
z(h)=Z(h) / Z\left(h_{0}\right)
$$

where $Z$ is in linear units; $h$ is the height of radar beam center. The $h_{0}$ is assumed as $1.5 \mathrm{~km}$ below the freezing level. As Fig. 3 shows, climatological VPRs are classified by different seasons and near-surface reflectivity values in $\mathrm{dBZ}$.

\section{Procedure for Real-Time Implementation}

The procedure of real-time climatology-based VPR correction is shown in Fig. 4. Since climatological VPRs have been quantified for S-band, the Ku-to-S-band conversion is no longer required for real-time processing. However, given a specific location, the appropriate climatological VPR needs to be determined for constructing the representative VPR. First, the current VPR-IE system only corrects the VPR for stratiform precipitation. The identification of stratiform is mainly based on the real-time NMQ product of precipitation type. Note that BB contamination sometimes can be misidentified as convective precipitation by NMQ. As a result, the VPR-IE system further examines the radar beam height and the freezing level to reduce this uncertainty. Secondly, the climatological VPRs have been quantified with the near-surface reflectivity, which correspond

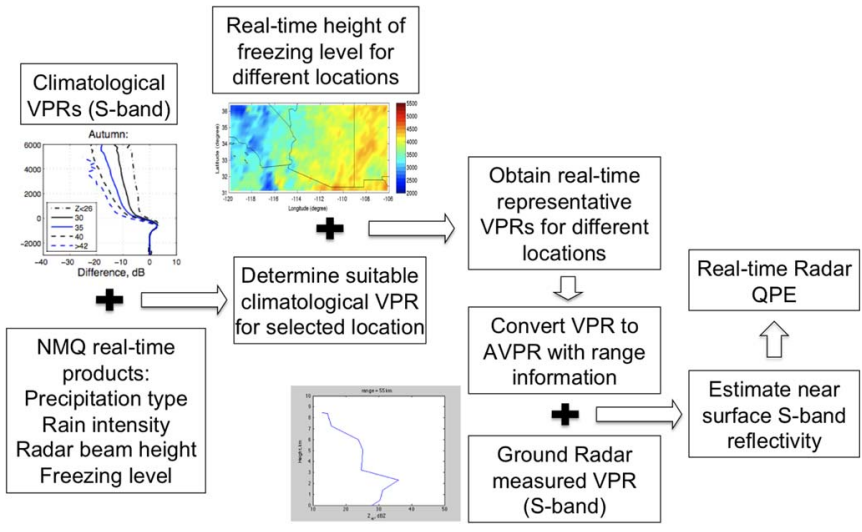

Fig. 4. Procedure of real-time climatology-based VPR correction.

to different rain intensities. The VPR-IE system applies a selfconsistency method, i.e., estimating the near-surface reflectivity with all the climatological VPRs and then checking if the result is consistent with the assumed reflectivity category. The selected climatological VPR is then combined with the realtime freezing level height to take into account the local variation of a storm as well as the underlying terrain. Considering the low revisit frequency of the TRMM satellite, the real-time freezing level data are adapted from NMQ. The VPR-IE system also updates the freezing level whenever the TRMM satellite passes over the region. Combining climatological VPRs and real-time freezing level data provides representative VPRs for different locations. Considering different radar ranges, representative VPRs are converted to the AVPRs, which are combined with ground-radar measurements to estimate the near-surface reflectivity. Consequently, the near-surface QPE can mitigate the underestimation (or overestimation) caused by pristine ice (or melting ice) signals in original ground-radar measurements. Although the spatial pattern of a VPR in an event may be different from the result of long-term statistics, the current VPR-IE system has ignored the spatial variability of VPRs and generally relies on the shape of the climatological VPR and the spatial pattern of the real-time freezing level height.

\section{CASE ANALYSis}

To demonstrate the performance of the developed VPR-IE system, this section shows a case study (22 January 2010) in AZ, where ground-radar coverage is limited by mountain blockage. This event is a winter stratiform storm that moved onshore from the Pacific Ocean and passed over the southwestern U.S. from west to east. The freezing level was close to the surface and snowfall was mainly reported in UT, NV, and northern CA.

The NMQ system provides a mosaicked hybrid scan reflectivity (HSR) product, which denotes the reflectivity observed by NEXRAD radars at the lowest available scanning height [17]. Fig. 5 shows the height of HSR (HSRH) and the terrain altitude in the analysis region $\left(32^{\circ} \mathrm{N}-37^{\circ} \mathrm{N}\right.$ and $\left.115^{\circ} \mathrm{W}-109^{\circ} \mathrm{N}\right)$. It is apparent that most HSR values have been measured aloft, not near the surface. It should be noted that the Phoenix and Yuma radars (KIWA and KYUX) were not available in realtime during this particular case due to a data outage.

Fig. 6(a) shows the HSR image (0350UTC). If this image were measured near the surface, it would imply that the storm 


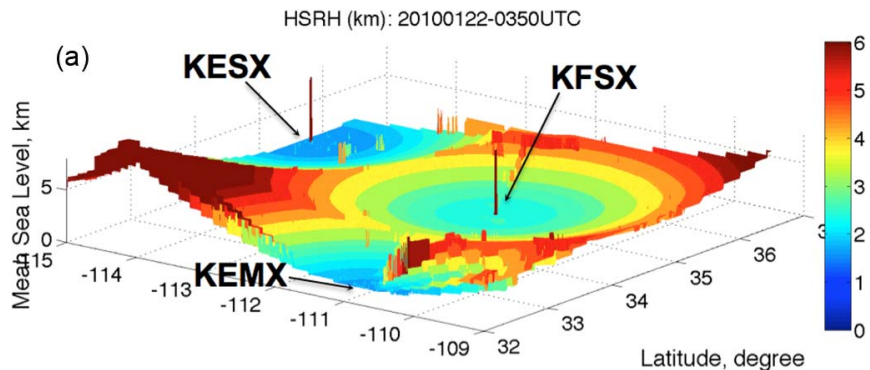

Longitude, degree

(b) Colorado River Grand Canyon

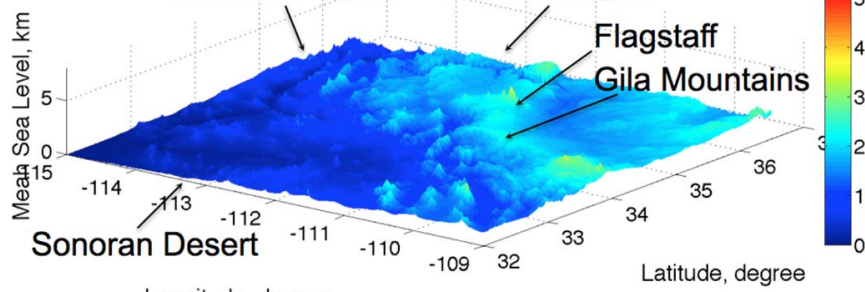

Longitude, degree

Fig. 5. (a) HSRH $(\mathrm{km})$ : the height of radar beam. (b) Terrain altitude (km).

Hybrid Scan Reflectivity (dBZ): 20100122-0350UTC

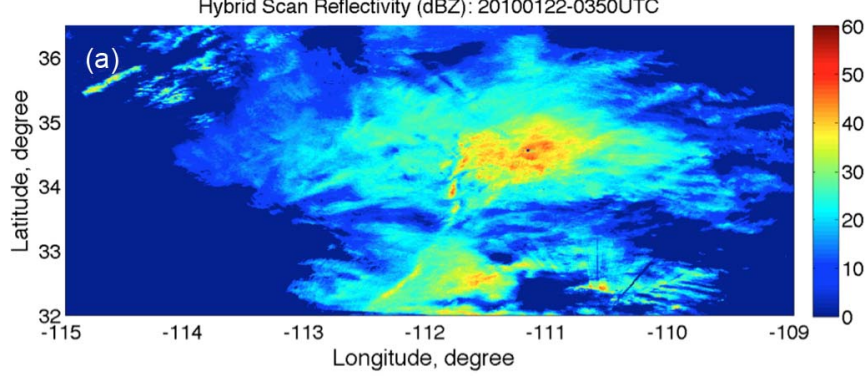

Hybrid Scan Reflectivity (dBZ): 20100122-0350UTC

(b)

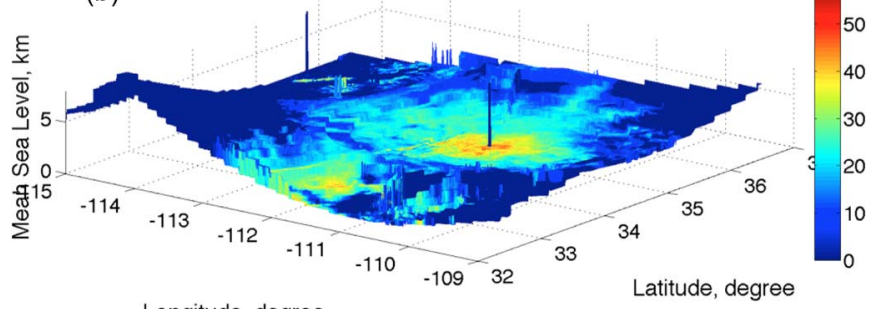

Longitude, degree

Fig. 6. (a) Hybrid scan reflectivity (HSR, unit in dBZ). (b) HSR plotted at the height of radar beam. (0359UTC, 22 January 2010).

core would be likely convective near the KFSX radar site. Low HSR values in the surrounding region suggest weak precipitation. As shown in Fig. 6(b), however, the HSR values have generally been measured within or above the melting layer, given the $0{ }^{\circ} \mathrm{C}$ isotherm is generally $2.5 \mathrm{~km}$ above mean sea level (MSL) in the analysis region. Because the melting layer is close to the surface, the apparent "storm core" near the KFSX site (2.26 km above MSL) is actually contamination from the BB signal. The "weak precipitation" in the surrounding regions is attributed to sampling in the ice region because the radar beam has overshot the melting layer.

In this case study, the climatological VPRs for stratiform precipitation during the winter are applied to estimate the near-surface precipitation following the procedure described in the previous section. It is noted that the observed melting layer in a local storm might have different heights due to
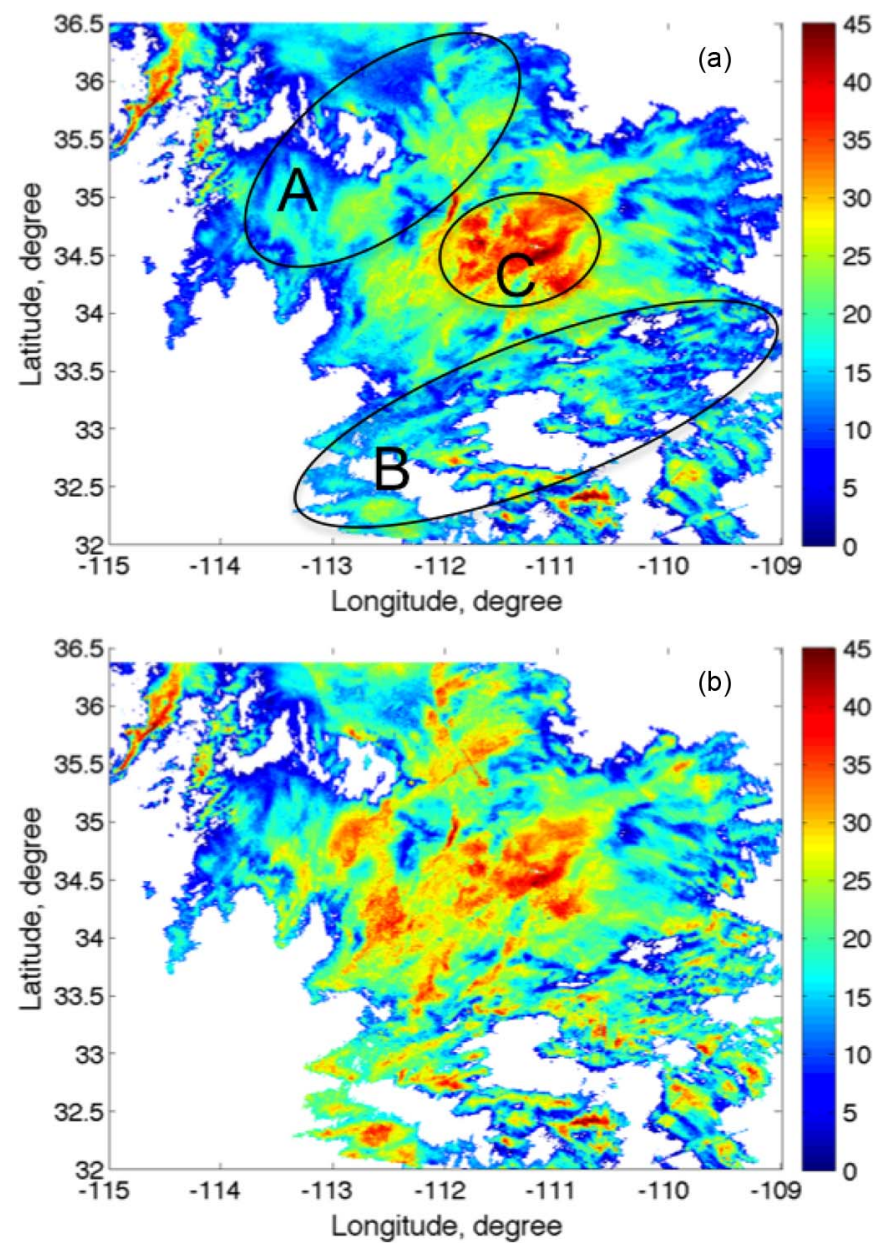

Fig. 7. Radar reflectivity (0235UTC, 22 January 2010) before (a) and after (b) the VPR-IE correction.

the VPR variation. According to instantaneous measurements of TRMM-PR during two passes at 0235UTC and 0410UTC, the freezing level difference between statistical results and instantaneous results is not substantial. The biases (or standard deviations) are $-92.3 \mathrm{~m}(377.6 \mathrm{~m})$ and $-174.4 \mathrm{~m}(436.5 \mathrm{~m})$ for these two passes, respectively. In the VPR-IE system, the height of the representative VPR is primarily determined based on the freezing level from NMQ system and adjusted with TRMM observations. That is to say, the bias is added into the representative VPR when TRMM passes over the region.

Fig. 7 illustrates an example of the VPR-IE correction. Fig. 7(a) shows reflectivity observed by NEXRAD radars on 22 January 2010 when a widespread stratiform precipitation passed the region of AZ. According to radar locations and the height of the radar beams, it is apparent that region $\mathrm{C}$ in Fig. 7(a) is contaminated by the BB signal. Regions A and B are related to measurements in the ice region. The corresponding QPE based on the reflectivity in Fig. 7(a) will overestimate (or underestimate) the rainfall in region $\mathrm{C}$ (or regions $\mathrm{A}$ and B). As shown in Fig. 7(b), the VPR-IE correction gives a more reasonable radar reflectivity image representative of nearsurface rainfall. The reflectivity has been reduced in region $\mathrm{C}$ and increased in regions A and B. The whole image in the analysis region reveals reflectivity patterns consistent with widespread stratiform precipitation. 
The VPR-IE system applies the same empirical $R-Z$ relations for QPE as is done in the NMQ system [17]. The stratiform relation $Z=200 R^{1.6}$ has been applied where $Z$ is the reflectivity in $\mathrm{mm}^{6} \mathrm{~m}^{-3}$ and $R$ is rainfall rate in $\mathrm{mm} \cdot \mathrm{h}^{-1}$. The QPE results before and after the VPR-IE correction have been compared with rain gauge measurements. We have examined radar QPE results from 0000UTC to 0500UTC in this event. One-hour rainfall accumulation has been computed and compared with the HADS (www.weather.gov/oh/hads/) hourly rain gauge data. The comparison results show a clear improvement of VPR-IE method over the unadjusted NEXRAD radar QPE. Before the correction, the radar QPE had a bias of $-3.32 \mathrm{~mm}$ and a root mean squared error (RMSE) of $5.47 \mathrm{~mm}$. After applying the VPR-IE correction, the bias has been improved to $-2.17 \mathrm{~mm}$ and the RMSE has been reduced to $4.88 \mathrm{~mm}$. Qualitatively speaking, the reflectivity images show much less dependence on the height of the radar beam relative to the melting layer.

\section{Discussion And CONCLUSION}

The case study demonstrates VPR-IE's potential of enhancing QPE in complex terrain where QPE is often degraded by signals from the ice region and/or melting layer. One potential error source of VPR-IE may be the VPR variability within a local storm, i.e., the deviation from the climatological VPR. We have adjusted the the height of freezing level observed with the system. There are still deviations related to BB width, VPR slope, and spatial variability, which are more difficult to adjust. Another potential error source is precipitation type identification. Different climatological VPRs are required for different types (stratiform/convection). However, the type identification in the NMQ system is unreliable when radar measurements are contaminated. Incorrect identification may lead to an erroneous correction factor applied. Moreover, the model errors associated with the climatological VPRs and the VPR conversion may cause QPE errors for the VPR-IE method. All these error sources, however, are not as significant as the contamination from the BB or ice signals. Therefore, the VPRIE is particularly useful in mountainous regions and/or at far range from radar.

In conclusion, a real-time VPR-IE system is being constructed at OU. This study introduces the basic structure of this system and summarizes its major procedure to estimate the near-surface reflectivity and subsequent QPE using the characterized VPR. Currently a near-real-time demo system of VPRIE has been developed in the Hydrometeorology \& Remote Sensing Laboratory at OU and can be visited from the website http://eos.ou.edu/NASA_SPoRT_DemoSystem.html. The demo system generates the near-real-time VPR-IE QPE products (update interval less than 5 minutes), which can be accessed from http://hydro.ou.edu/NASA_Data/SPoRT_Data/. The successful implementation of the proposed VPR-IE system will help to incorporate the NMQ system and TRMMPR products in an operational QPE system, which covers the whole CONUS. Systematic evaluation of the VPR-IE method using the near-real-time demo system will be addressed in forthcoming studies.

\section{ACKNOWLEDGMENT}

The authors are grateful of Dr. J. Zhang from NSSL, who has provided expertise in the NMQ system as well as data sets. The authors also appreciate scientists and engineers of NASA's TRMM mission for making TRMM products available.

\section{REFERENCES}

[1] R. A. Maddox, J. Zhang, J. J. Gourley, and K. W. Howard, "Weather radar coverage over the contiguous United States," Weather Forecast., vol. 17, no. 4, pp. 927-934, Aug. 2002.

[2] F. Fabry and I. Zawadzki, "Long-term radar observations of the melting layer of precipitation and their interpretation," J. Atmos. Sci., vol. 52, no. 7, pp. 838-851, Apr. 1995.

[3] Q. Cao, Y. Hong, J. J. Gourley, Y. Qi, J. Zhang, Y. Wen, and P. Kirstetter, "Statistical and physical analysis of vertical structure of precipitation in mountainous west region of US using 11+ year spaceborne TRMM PR observations," J. Appl. Meteor. Climatol., vol. 52, no. 2, pp. 408-424, Feb. 2013.

[4] B. Vignal, H. Andrieu, and J. Dominique Creutin, "Identification of vertical profiles of reflectivity from volume scan radar data," J. Appl. Meteor. vol. 38, no. 8, pp. 1214-1228, Aug. 1999.

[5] J. J. Gourley and C. M. Calvert, "2003: Automated detection of the bright band using WSR-88D data," Weather Forecast., vol. 18, no. 4, pp. 585-599, Aug. 2003.

[6] P. Tabary, "The new french operational radar rainfall product. Part I: Methodology," Weather Forecast., vol. 22, no. 3, pp. 393-408, Jun. 2007.

[7] S. Y. Matrosov, K. A. Clark, and D. E. Kingsmill, "A polarimetric radar approach to identify rain, melting-layer, and snow regions for applying corrections to vertical profiles of reflectivity," J. Appl. Meteor. Climatol., vol. 46, no. 2, pp. 154-166, Feb. 2007

[8] J. Zhang, C. Langston, and K. Howard, "Brightband identification based on vertical profiles of reflectivity from the WSR-88D," J. Atmos. Oceanic Technol., vol. 25, no. 10, pp. 1859-1872, Oct. 2008.

[9] J. Zhang and Y. Qi, "A real-time algorithm for the correction of brightband effects in radar-derived QPE," J. Hydrometeor, vol. 11, no. 5, pp. $1157-$ 1171, Oct. 2010.

[10] Y. Qi, J. Zhang, and P. Zhang, "A real-time automated convective and stratiform precipitation segregation algorithm in native radar coordinates," Q. J. R. Meteorol. Soc., vol. 139, no. 677, pp. 2233-2240, Oct. 2013.

[11] M. N. Anagnostou, J. Kalogiros, E. N. Anagnostou, M. Tarolli, A. Papadopoulos, and M. Borga, "Performance evaluation of highresolution rainfall estimation by X-band dual-polarization radar for flash flood applications in mountainous basins," J. Hydrology, vol. 394, no. 1/2, pp. 4-16, Nov. 2010.

[12] J. Kalogiros, M. N. Anagnostou, E. N. Anagnostou, M. Montopoli, E. Picciotti, and F. S. Marzano, "Correction of polarimetric radar reflectivity measurements and rainfall estimates for apparent vertical profile in stratiform rain," J. Appl. Meteor. Climatol., vol. 52, no. 5, pp. 1170-1186, May 2013.

[13] Y. Wen, Q. Cao, P. Kirstetter, Y. Hong, J. J. Gourley, J. Zhang, G. Zhang, and B. Yong, "Incorporating NASA space-borne radar data into NOAA National Mosaic QPE system for improved precipitation measurement: A physically based VPR identification and enhancement method,' J. Hydrometeor, vol. 14, no. 4, Aug. 2013.

[14] Q. Cao, Y. Hong, Y. Qi, Y. Wen, J. Zhang, J. Gourley, and L. Liao, "Empirical conversion of vertical profile of reflectivity (VPR) from $\mathrm{Ku}$ band to S-band frequency," J. Geophys. Res. Atmos., vol. 118, no. 4, pp. 1814-1825, Feb. 2013.

[15] T. Kozu, T. Kawanishi, H. Kuroiwa, M. Kojima, K. Oikawa, H. Kumagai, K. Okamoto, M. Okumura, H. Nakatsuka, and K. Nishikawa, "Development of precipitation radar onboard the Tropical Rainfall Measuring Mission (TRMM) satellite," IEEE Trans. Geosci. Remote Sens., vol. 39, no. 1, pp. 102-116, Jan. 2001.

[16] J. Awaka, T. Iguchi, and K. Okamoto, “TRMM PR standard algorithm 2A23 and its performance on bright band detection," J. Meteor. Soc. Jpn., vol. 87A, pp. 31-52, 2009.

[17] J. Zhang, K. Howard, C. Langston, S. Vasiloff, B. Kaney, A. Arthur, S. Van Cooten, K. Kelleher, D. Kitzmiller, F. Ding, D.-J. Seo, E. Wells, and C. Dempsey, "National mosaic and multi-sensor QPE (NMQ) System: Description, results, and future plans," Bull. Amer. Meteor. Soc., vol. 92, no. 10 , pp. 1321-1338, Oct. 2011. 